\title{
Price of Anarchy for Polynomial Wardrop Games *
}

\author{
Dominic Dumrauf and Martin Gairing \\ Faculty of Computer Science, Electrical Engineering and Mathematics, \\ University of Paderborn, Fürstenallee 11, 33102 Paderborn, Germany. \\ \{masa, gairing\} @uni-paderborn. de
}

(August 15, 2006)

\begin{abstract}
In this work, we consider Wardrop games where traffic has to be routed through a shared network. Traffic is allowed to be split into arbitrary pieces and can be modeled as network flow. For each edge in the network there is a latency function that specifies the time needed to traverse the edge given its congestion. In a Wardrop equilibrium, all used paths between a given source-destination pair have equal and minimal latency.

In this paper, we allow for polynomial latency functions with an upper bound $d$ and a lower bound $s$ on the degree of all monomials that appear in the polynomials. For this environment, we prove upper and lower bounds on the price of anarchy.
\end{abstract}

\section{Introduction}

Motivation and Framework. The price of anarchy, also known as coordination ratio, has been defined in the seminal work by Koutsoupias and Papadimitriou [14] as a measure of the extent to which competition approximates cooperation. In general, the price of anarchy is the worst-case ratio between the value of a social objective function, usually coined as social cost, in some equilibrium state of a system, and that of some social optimum. Usually, the equilibrium state has been taken to be that of a Nash equilibrium [16] - a state in which no user wishes to unilaterally leave its own strategy in order to improve the value of its private objective function, also known as individual cost. So, the price of anarchy represents a rendezvous of Nash equilibrium, a concept fundamental to Game Theory, with approximation, an ubiquitous concept in Theoretical Computer Science today (see, e.g., [22]).

The Wardrop model has already been studied in the context of road traffic systems by Pigou [17] in the 1920's and later by Wardrop [23], and by Beckmann, McGuire and Winsten [3] in the 1950's. For a survey of the early work

\footnotetext{
* This work has been partially supported by the DFG-SFB 376 and by the European Union within the Integrated Project IST-15964 "Algorithmic Principles for Building Efficient Overlay Computers" (AEOLUS).
} 
on this model see [4]. In the Wardrop model, traffic has to be sent through a shared network and traffic is allowed to be split into arbitrary pieces. In this environment, unregulated traffic is modeled as network flow. Wardrop [23] introduced the concept of Wardrop equilibrium to describe user behavior in this kind of traffic networks. Given an arbitrary network with edge latency functions, Wardrop equilibria have been classified as flows with all flow paths used between a given source-destination pair having equal latency. A Wardrop equilibrium can be interpreted as a Nash equilibrium in a game with infinitely many users, each carrying an infinitesimal amount of traffic from a source to a destination.

Inspired by the arisen interest in the price of anarchy, Roughgarden and Tardos [21] re-investigated the Wardrop model and used the total latency as their social objective function. The total latency is a measure for the total travel time. In this context, the exact value for the price of anarchy was shown for linear latency functions by Roughgarden and Tardos [21] and for arbitrary polynomial latency functions with nonnegative coefficients and maximum degree $d$ by Roughgarden [19]. In his book [20, Chapter 3], Roughgarden gives the following rule of thumb:

The price of anarchy is small unless cost functions are extremely steep.

In this work, we examine this rule of thumb closer by re-considering the price of anarchy for polynomial latency functions of maximum degree $d$. However, in contrast to the latency functions considered by Roughgarden [19], our latency functions have also a minimum degree of $s$. For large $d$ these latency functions are extremely steep, however, we show that in many cases the price of anarchy remains small.

Related Work. The price of anarchy was introduced by Koutsoupias and Papadimitriou [14] and received a lot of attention in various routing games (see e.g. $[1,2,5-10,12,13,15,19,21])$.

Early work on the Wardrop model has been done in the context of road traffic systems $[3,4,17,23]$. Beckmann et al. [3] showed that a Wardrop equilibrium always exists and that it is essentially unique. These results were based on the observation that a Wardrop equilibrium is a solution to a related convex program.

For the Wardrop model, with social cost as total latency, Roughgarden and Tardos [21] showed that the price of anarchy is exactly $\frac{4}{3}$ in case of linear latency functions. For the case of polynomial latency functions of maximum degree $d$, Roughgarden [19] showed that the price of anarchy is $\frac{(d+1) \sqrt[d]{d+1}}{(d+1) \sqrt[d]{d+1}-d}$. Interestingly, in both cases, the price of anarchy is independent of the network topology, as it is achieved on the simple network of two parallel links [19,21]. Correa et al. [6] improved the bounds from [19] on the price of anarchy for the special case of polynomial latency functions without constant term and for $d \leq 4$. The price of anarchy was also studied for latency functions that arise as delay functions of $\mathrm{M} / \mathrm{M} / 1$ queues [19]. For arbitrary nondecreasing latency functions Roughgarden and Tardos [21] showed that the total latency in a Wardrop equilibrium is upper bounded by the optimum total latency for the instance where all traffic demands are doubled. 
Related to Wardrop games are (weighted) congestion games as introduced by Rosenthal [18]. In a congestion game, there is a set of resources and players can choose as their strategy a set of resources from a given set of subsets of resources. Awerbuch et al. [2] and Christodoulou and Koutsoupias [5] were the first to study the price of anarchy for congestion games. They showed asymptotic tight bounds on the price of anarchy for congestion games with polynomial latency functions in case of unweighted $[2,5]$ and in case of weighted player demands [2]. With a more careful analysis, Aland et al. [1] were able to derive the exact value for the price of anarchy in both cases. For a survey on weighted congestion games, we refer to [11].

Contribution. In this paper, we study the price of anarchy for Wardrop games with polynomial latency functions in more detail. In particular, we consider polynomials that consist of monomials of maximum degree $d$ and minimum degree $s$. All our latency functions have nonnegative coefficients. We will call such polynomials $(d, s)$-polynomials.

As our first result, we show that for general $(d, s)$-polynomials, the price of anarchy $(\operatorname{PoA}(\mathrm{d}, \mathrm{s}))$ is upper bounded by

$$
\operatorname{PoA}(d, s) \leq \frac{\left(\frac{d}{d+1}\right)^{d}}{(d+1) \cdot\left(\frac{\left(\frac{d}{d+1}\right)^{d}(s+1)}{\left(\frac{s}{s+1}\right)^{s}(d+1)}\right)^{\frac{d}{d-s}} \cdot\left(1-\left(\frac{\left(\frac{d}{d+1}\right)^{d}(s+1)}{\left(\frac{s}{s+1}\right)^{s}(d+1)}\right)^{\frac{1}{d-s}}\right)} .
$$

To achieve this result, we adopt a technique that was already used in [1] and that is again based on a technique from [5]. The core of our analysis is to determine parameters $c_{1}$ and $c_{2}$, such that

$$
y \cdot f(z) \leq c_{1} \cdot z \cdot f(z)+c_{2} \cdot y \cdot f(y)
$$

for all $(d, s)$-polynomials $f$ and for all reals $y, z \geq 0$. Table 1 shows numerical values for the upper bound for all $(d, s)$-polynomials with $d \leq 10$. The values for

\begin{tabular}{|c|c|c|c|c|c|c|c|c|c|}
\hline$s \backslash^{d}$ & 112 & 3 & 4 & 5 & 6 & 7 & 8 & 9 & 10 \\
\hline$\overline{0}$ & $\begin{array}{ll}\frac{4}{3} & 1.62575\end{array}$ & 1.89563 & 2.15050 & 2.39438 & 2.62971 & 2.85814 & 3.08084 & $=3.29856$ & 3.51206 \\
\hline 1 & 1.03551 & 1.09820 & 1.16756 & 1.23859 & 1.30962 & 1.38002 & 1.44954 & 1.51811 & 1.58575 \\
\hline 2 & & 1.01466 & 1.04498 & 1.08174 & 1.12147 & 1.16262 & 1.20439 & 1.24638 & 1.28834 \\
\hline 3 & & & 1.00805 & 1.02614 & 1.04938 & 1.07547 & 1.10324 & 1.13199 & 1.16131 \\
\hline 4 & & & & 1.00510 & 1.01717 & 1.03329 & 1.05192 & 1.07217 & 1.09348 \\
\hline 5 & & & & & 1.00352 & 1.01215 & 1.02404 & $=1.03808$ & 1.05358 \\
\hline 6 & & & & & & 1.00257 & 1.00907 & 1.01821 & 1.02918 \\
\hline 7 & & & & & & & 1.00197 & 1.00703 & 1.01428 \\
\hline 8 & & & & & & & & 1.00155 & 1.00561 \\
\hline 9 & & & & & & & & & 1.00125 \\
\hline
\end{tabular}
$s=0$ have already been shown by Roughgarden [19]. Observe that the values

Table 1. Example values for our upper bound on the price of anarchy 
for $s=1$ and $d \leq 4$ improve the upper and match the lower bounds from [6].

We then prove monotonicity results on our upper bound. In particular, we show that the upper bound on $\operatorname{PoA}(d, s)$ is monotone increasing in $d$ and decreasing in $s$. Furthermore, we show that if $s=\frac{d}{a}$ is a constant fraction of $d$, then the upper bound on $\operatorname{PoA}\left(d, \frac{d}{a}\right)$ is still monotone increasing in $d$. Equipped with these results, we apply the limit for $d \rightarrow \infty$ to prove that for any $a>1$, $\operatorname{PoA}\left(d,\left\lceil\frac{d}{a}\right\rceil\right)$ is upper bounded by a constant. More precisely, we show that

$$
\operatorname{PoA}\left(d,\left\lceil\frac{d}{a}\right\rceil\right) \leq \frac{a^{\frac{1}{a-1}} \cdot(a-1)}{\mathrm{e} \cdot \ln (a)} .
$$

For instance, this gives upper bounds of 1.0614756 and 1.159983 for $a=2$ and $a=3$, respectively.

We close our paper with a discussion on lower bounds on the price of anarchy for Wardrop games with $(d, s)$-polynomials. Here, we use the very simple network of two parallel links. So far, we could not show that our general upper bound yields the exact value for the price of anarchy; however, numerical analysis for all $(d, s)$-polynomials with $d \leq 30$ gives a strong indication that this is the case.

For sufficiently large $d$ and for the cases $s=\frac{d}{2}$ and $s=\frac{d}{3}$, we give almost matching lower bounds on $\operatorname{PoA}(d, s)$.

Roadmap. The rest of this paper is organized as follows. Section 2 introduces the Wardrop model. Section 3.1 presents the upper bound on the price of anarchy, whereas Section 3.2 discusses lower bounds. We conclude in Section 4 with a summary of our results and some open problems. Due to lack of space, we omit some proofs. They can be found in the appendix.

\section{Notation}

For all $k \in \mathbb{N}$ denote $[k]=\{1, \ldots, k\}$.

Routing with Splittable Traffic. A Wardrop game is a tuple $\Gamma=(n, G, \mathrm{w}, \mathcal{P}, \mathbf{f})$. Here, $n$ is the number of players and $G=(V, E)$ is an undirected (multi)graph. The vector $\mathbf{w}=\left(w_{1}, \ldots, w_{n}\right)$ defines for every player $i \in[n]$ its traffic $w_{i} \in \mathbb{R}^{+}$. For each player $i \in[n]$ the set $\mathcal{P}_{i} \subset 2^{E}$ consists of all possible routing paths in $G=(V, E)$ from some node $s_{i} \in V$ to some other node $t_{i} \in V$. Denote $\mathcal{P}=\mathcal{P}_{1} \times \ldots \times \mathcal{P}_{n}$. Denote by $\mathrm{f}=\left\{f_{e} \mid e \in E\right\}$ the set of differentiable, monotone increasing and nonnegative edge latency functions.

In this paper, we allow for polynomial latency functions with nonnegative coefficients, where monomials of degree less than $s$ are missing; that is, latency functions are of the form $f_{e}(x)=\sum_{i=s}^{d} a_{i e} x^{i}$ with $a_{i e} \geq 0$ for all integers $s \leq i \leq d$ and all edges $e \in E$. We will call such latency functions $(d, s)$-polynomials.

Strategies and Strategy Profiles. A player $i \in[n]$ can split its traffic $w_{i}$ over the paths in $\mathcal{P}_{i}$. A strategy for player $i \in[n]$ is a tuple $\mathrm{x}_{i}=\left(x_{i P_{i}}\right)_{P_{i} \in \mathcal{P}_{i}}$ with $\sum_{P_{i} \in \mathcal{P}_{i}} x_{i P_{i}}=w_{i}$ and $x_{i P_{i}} \geq 0$ for all $P_{i} \in \mathcal{P}_{i}$. Denote by $\mathcal{X}_{i}=\left\{\mathrm{x}_{i} \mid \mathrm{x}_{i}\right.$ is a strategy for player $i\}$ the set of all strategies for player $i$. A strategy profile $\mathrm{x}=$ $\left(\mathrm{x}_{1}, \ldots, \mathrm{x}_{n}\right)$ is an $n$-tuple of strategies for the players. Define $\mathcal{X}=\mathcal{X}_{1} \times \ldots \times \mathcal{X}_{n}$ as the set of all possible strategy profiles. 
Wardrop Equilibria. For a strategy profile $\mathrm{x}$, the load $l_{e}(\mathrm{x})$ on an edge $e \in E$ is given by $l_{e}(\mathrm{x})=\sum_{i \in[n]} \sum_{P_{i} \in \mathcal{P}_{i}, P_{i} \ni e} x_{i P_{i}}$. A strategy profile $\mathrm{x}$ is a Wardrop equilibrium, if for every player $i \in[n]$, and every $P_{i}, P_{i}^{\prime} \in \mathcal{P}_{i}$ with $x_{i P_{i}}>0$ it holds that

$$
\sum_{e \in P_{i}} f_{e}\left(l_{e}(\mathrm{x})\right) \leq \sum_{e \in P_{i}^{\prime}} f_{e}\left(l_{e}(\mathrm{x})\right) .
$$

Observe that in a Wardrop equilibrium all flow paths of a player have equal latency. We can regard each player $i \in[n]$ as a service provider who has many clients each handling a negligible small amount of traffic. In a Wardrop equilibrium, each service provider satisfies all his clients because none of them can improve its experienced latency.

Social Cost and Price of Anarchy. For a strategy profile $x$, define the social cost $\mathrm{SC}(\mathrm{x})$ as the total latency; thus,

$$
\mathrm{SC}(\mathrm{x})=\sum_{i \in[n]} \sum_{P_{i} \in \mathcal{P}_{i}} x_{i P_{i}} \sum_{e \in P_{i}} f_{e}\left(l_{e}(\mathrm{x})\right) .
$$

This social cost is motivated by the interpretation as a game with infinitely many players with negligible demand and models the sum of the players latencies. The optimum associated with a game is defined by OPT $=\min _{x \in \mathcal{X}} \mathrm{SC}(\mathrm{x})$. The price of anarchy, also called coordination ratio and denoted $\mathrm{PoA}$, is the maximum value, over all instances and Wardrop equilibria $x$, of the ratio $\frac{\mathrm{SC}(\mathrm{x})}{\mathrm{OPT}}$. For the class of Wardrop games, where all latency functions are $(d, s)$-polynomials, denote by $\operatorname{PoA}(d, s)$ the price of anarchy with respect to $d$ and $s$.

\section{Price of Anarchy}

\subsection{Upper Bound}

Before proving a general upper bound on the price of anarchy for Wardrop games with $(d, s)$-polynomial latency functions, we have to prove the following technical lemma:

Lemma 1. Let $s, d \in \mathbb{N}$ with $s \leq d$. Choose $c_{1}, c_{2} \in \mathbb{R}_{\geq 0}$ such that

$$
\begin{array}{lll}
y \cdot z^{s} \leq c_{1} \cdot z^{s+1}+c_{2} \cdot y^{s+1} & \forall y, z \in \mathbb{R}_{>0} \\
\text { and } \quad y \cdot z^{d} \leq c_{1} \cdot z^{d+1}+c_{2} \cdot y^{d+1} & \forall y, z \in \mathbb{R}_{>0} .
\end{array}
$$

Then, it follows that

$$
y \cdot z^{i} \leq c_{1} \cdot z^{i+1}+c_{2} \cdot y^{i+1} \quad \forall i \in \mathbb{N}: s \leq i \leq d \forall y, z \in \mathbb{R}_{>0} .
$$

Proof. Since

$$
y \cdot z^{i} \leq c_{1} \cdot z^{i+1}+c_{2} \cdot y^{i+1} \quad \forall i \in \mathbb{N}: s \leq i \leq d \forall y, z \in \mathbb{R}_{>0}
$$

is equivalent to

$$
\left(\frac{z}{y}\right)^{i} \leq c_{1} \cdot\left(\frac{z}{y}\right)^{i+1}+c_{2} \quad \forall i \in \mathbb{N}: s \leq i \leq d \forall y, z \in \mathbb{R}_{>0}
$$


it suffices to show that

$$
z^{i} \leq c_{1} \cdot z^{i+1}+c_{2} \quad \forall i \in \mathbb{N}: s \leq i \leq d \forall z \in \mathbb{R}_{>0} .
$$

This follows by replacing $\frac{z}{y} \in \mathbb{R}_{>0}$ with a new $z \in \mathbb{R}_{>0}$. Furthermore, it follows from (1) and (2) that

$$
\begin{aligned}
& z^{s} \leq c_{1} \cdot z^{s+1}+c_{2} & \forall z \in \mathbb{R}_{>0} \\
\text { and } \quad & z^{d} \leq c_{1} \cdot z^{d+1}+c_{2} & \forall z \in \mathbb{R}_{>0} .
\end{aligned}
$$

Fix an arbitrary $i \in \mathbb{N}$ with $s \leq i \leq d$. We proceed by case study dependent on $z \in \mathbb{R}_{>0}$.

First assume that $z \leq 1$. Let $i=s+j$, then $0 \leq j \leq d-s$. We get

$$
z^{i}=z^{s+j} \leq z^{j}\left(c_{1} \cdot z^{s+1}+c_{2}\right)=c_{1} z^{i+1}+z^{j} \cdot c_{2} \leq c_{1} z^{i+1}+c_{2},
$$

since $z \leq 1$.

Now assume that $z \geq 1$. Let $i=d-j$, then $0 \leq j \leq d-s$. We get

$$
z^{i}=z^{d-j} \leq \frac{1}{z^{j}}\left(c_{1} \cdot z^{d+1}+c_{2}\right)=c_{1} z^{i+1}+\frac{1}{z^{j}} \cdot c_{2} \leq c_{1} z^{i+1}+c_{2},
$$

since $z \geq 1$.

In any case $z^{i} \leq c_{1} \cdot z^{i+1}+c_{2}$. This completes the proof of the lemma.

We are now ready to prove our general upper bound on the price of anarchy.

Theorem 1. For Wardrop games with $(d, s)$-polynomial latency functions, we have

$$
\operatorname{PoA}(d, s) \leq \frac{\left(\frac{d}{d+1}\right)^{d}}{(d+1) \cdot\left(\frac{\left(\frac{d}{d+1}\right)^{d}(s+1)}{\left(\frac{s}{s+1}\right)^{s}(d+1)}\right)^{\frac{d}{d-s}} \cdot\left(1-\left(\frac{\left(\frac{d}{d+1}\right)^{d}(s+1)}{\left(\frac{s}{s+1}\right)^{s}(d+1)}\right)^{\frac{1}{d-s}}\right)} .
$$

Proof. Observe, that for $s=0$ our upper bound on the price of anarchy reduces to the exact value on the price of anarchy that was proved by Roughgarden [19] for this case. So, in the following, we assume that $s \geq 1$. Let $\mathrm{x}=\left(x_{1 P_{1}}, \ldots, x_{n P_{n}}\right)$ be a Wardrop equilibrium and let $\mathrm{x}^{*}=\left(x_{1 P_{1}}^{*}, \ldots, x_{n P_{n}}^{*}\right)$ be a strategy profile with optimum social cost. Since $\mathrm{x}$ is a Wardrop equilibrium, it follows by definition of a Wardrop equilibrium that

$$
\begin{aligned}
\mathrm{SC}(\mathrm{x}) & =\sum_{i \in[n]} \sum_{P_{i} \in \mathcal{P}_{i}} x_{i P_{i}} \sum_{e \in P_{i}} f_{e}\left(l_{e}(\mathrm{x})\right) \leq \sum_{i \in[n]} \sum_{P_{i} \in \mathcal{P}_{i}} x_{i P_{i}}^{*} \sum_{e \in P_{i}} f_{e}\left(l_{e}(\mathrm{x})\right) \\
& =\sum_{e \in E} \underbrace{l_{e}\left(\mathrm{x}^{*}\right)}_{:=y} \cdot \underbrace{f_{e}\left(l_{e}(\mathrm{x})\right)}_{:=x} .
\end{aligned}
$$

Now, since $l_{e}\left(\mathrm{x}^{*}\right)$ and $l_{e}(\mathrm{x})$ are both positive real numbers, assume that $c_{1}$ and $c_{2}$ are such that

$$
y \cdot f(z) \leq c_{1} \cdot z \cdot f(z)+c_{2} \cdot y \cdot f(y) \quad \forall x, y \in \mathbb{R}_{\geq 0}
$$


for all polynomials $f$ with minimum degree $s$ and maximum degree $d$, having nonnegative coefficients. Then,

$$
\begin{aligned}
\mathrm{SC}(\mathrm{x}) & \leq c_{1} \cdot \sum_{e \in E} l_{e}(\mathrm{x}) \cdot f_{e}\left(l_{e}(\mathrm{x})\right)+c_{2} \cdot \sum_{e \in E} l_{e}\left(\mathrm{x}^{*}\right) \cdot f_{e}\left(l_{e}\left(\mathrm{x}^{*}\right)\right) \\
& =c_{1} \cdot \mathrm{SC}(\mathrm{x})+c_{2} \cdot \mathrm{SC}\left(\mathrm{x}^{*}\right)
\end{aligned}
$$

and with $0<c_{1}<1$ it follows that

$$
\frac{\mathrm{SC}(\mathrm{x})}{\mathrm{SC}\left(\mathrm{x}^{*}\right)} \leq \frac{c_{2}}{1-c_{1}} .
$$

Since $\mathrm{x}$ is an arbitrary Wardrop equilibrium, we get

$$
\operatorname{PoA}(d, s) \leq \frac{c_{2}}{1-c_{1}} .
$$

We will now show how to determine $c_{1}$ and $c_{2}$ such that inequality (3) holds and that the resulting upper bound is minimal. In case $y=0$ equation (3) follows immediately from $c_{1} \geq 0$ and $z \geq 0$. In case $z=0$ the left hand side yields $y \cdot f(0)=0$, since by the degree of the lowest monomial being $s \geq 1$ there are no additive constants in the latency functions. This is always less or equal than $c_{2} \cdot y \cdot f(y)$, since latency functions are monotone increasing, $y \geq 0$ and $c_{2} \geq 0$. So, in the following we assume that $y>0$ and $z>0$. In order to show that (3) holds, it suffices to show that (3) holds for all monomials of degree $i \in[n]$ with $s \leq i \leq d$, since polynomials are a linear combination of monomials. This implies that (3) then holds also for the considered polynomials. By Lemma 1 it suffices to show this for the monomials of degree $s$ and $d$. Consider inequality (3) for a single monomial $f(z)=a_{i} \cdot z^{i}$, which we divide by $a_{i} \cdot y^{i+1}$ yielding

$$
\left(\frac{z}{y}\right)^{i} \leq c_{1} \cdot\left(\frac{z}{y}\right)^{i+1}+c_{2} \quad \forall s \leq i \leq d \forall y, z \in \mathbb{R}_{>0} .
$$

Set $\hat{z}:=\frac{z}{y}$. Then $\hat{z} \in \mathbb{R}_{>0}$ and (3) reduces to

$$
\hat{z}^{i} \leq c_{1} \cdot \hat{z}^{i+1}+c_{2} \quad \forall s \leq i \leq d \forall \hat{z} \in \mathbb{R}_{>0} .
$$

We now view (5) as a function in $i, c_{1}$ and $\hat{z}$, since we want to determine the maximum $\hat{z}$ such that inequality (3) holds. Thus, we have the following function

$$
c_{2}\left(i, c_{1}, \hat{z}\right):=\hat{z}^{i}-c_{1} \cdot \hat{z}^{i+1},
$$

which we partially differentiate in $\hat{z}$ in order to retrieve the minimum $c_{2}$ such that (5) holds, yielding

$$
\frac{\partial}{\partial \hat{z}} c_{2}\left(i, c_{1}, \hat{z}\right)=i \cdot \hat{z}^{i-1}-(i+1) \cdot c_{1} \cdot \hat{z}^{i} .
$$

The $\hat{z}$ for which $c_{2}$ is maximum can now be easily determined to be $\hat{z}^{\max }:=$ $\frac{i}{c_{1} \cdot(i+1)}$. Simple insertion in (6) yields

$$
\begin{aligned}
c_{2}\left(i, c_{1}, \hat{z}^{\max }\right) & =\left(\frac{i}{c_{1} \cdot(i+1)}\right)^{i}-c_{1} \cdot\left(\frac{i}{c_{1} \cdot(i+1)}\right)^{i+1}=\frac{(i+1) \cdot i^{i}-i^{i+1}}{(i+1)^{i+1} \cdot c_{1}^{i}} \\
& =\frac{\left(\frac{i}{i+1}\right)^{i}}{c_{1}^{i} \cdot(i+1)} .
\end{aligned}
$$


We define

$$
c_{2}\left(i, c_{1}\right):=\frac{\left(\frac{i}{i+1}\right)^{i}}{c_{1}^{i} \cdot(i+1)} .
$$

Lemma 1 states that it suffices to focus on the monomials of degree $s$ and $d$ in order for (3) to hold. We therefore determine $c_{1}$ as a solution to the equation of $c_{2}\left(d, c_{1}\right)=c_{2}\left(s, c_{1}\right)$. Thus, we have

$$
\frac{\left(\frac{d}{d+1}\right)^{d}}{c_{1}^{d} \cdot(d+1)}=\frac{\left(\frac{s}{s+1}\right)^{s}}{c_{1}^{s} \cdot(s+1)}
$$

or equivalently

$$
c_{1}=\left(\frac{(s+1) \cdot\left(\frac{d}{d+1}\right)^{d}}{(d+1) \cdot\left(\frac{s}{s+1}\right)^{s}}\right)^{\frac{1}{d-s}} .
$$

Having calculated $c_{1}$, we can retrieve $c_{2}$ by simple insertion in (7) using the maximum degree $d$ of the monomials yielding

$$
c_{2}:=c_{2}\left(d, c_{1}\right)=\frac{\left(\frac{d}{d+1}\right)^{d}}{\left(\frac{(s+1) \cdot\left(\frac{d}{d+1}\right)^{d}}{(d+1)\left(\frac{s}{s+1}\right)^{s}}\right)^{\frac{d}{d-s}} \cdot(d+1)} .
$$

We get with (4) that

$$
\begin{aligned}
\operatorname{PoA}(d, s) \leq \frac{c_{2}}{1-c_{1}}=\frac{\frac{\left(\frac{d}{d+1}\right)^{d}}{\left(\frac{(s+1) \cdot\left(\frac{d}{d+1}\right)^{d}}{(d+1)\left(\frac{s}{s+1}\right)^{s}}\right)^{\frac{d}{d-s}} \cdot(d+1)}}{1-\left[\frac{(s+1) \cdot\left(\frac{d}{d+1}\right)^{d}}{(d+1) \cdot\left(\frac{s}{s+1}\right)^{s}}\right]^{\frac{1}{d-s}}} \\
=\frac{\left(\frac{d}{d+1}\right)^{d}}{(d+1) \cdot\left(\frac{\left(\frac{d}{d+1}\right)^{d}(s+1)}{\left(\frac{s}{s+1}\right)^{s}(d+1)}\right)^{\frac{d}{d-s}} \cdot\left(1-\left(\frac{\left(\frac{d}{d+1}\right)^{d}(s+1)}{\left(\frac{s}{s+1}\right)^{s}(d+1)}\right)^{\frac{1}{d-s}}\right)},
\end{aligned}
$$

which completes the proof of the theorem.

Having proved the general upper bound, we now investigate the case $s=\left\lceil\frac{d}{a}\right\rceil$ with $a \in \mathbb{R}$ and $1 \leq a \leq d$. For the case $a=1$, we have that $\operatorname{PoA}(d, d)=1$ as shown in [21] for $d=1$ and Theorem 1 shows that this also holds for the case $d \geq 2$. In order to prove an upper bound on $\operatorname{PoA}\left(d,\left\lceil\frac{d}{a}\right\rceil\right)$, we first show monotonicity results for the upper bound from Theorem 1.

Lemma 2. The upper bound on $\operatorname{PoA}(d, s)$ from Theorem 1 is monotone decreasing in $s$. 
Lemma 3. The upper bound on $\operatorname{PoA}\left(d, \frac{d}{a}\right)$ from Theorem 1 is monotone increasing in $d$.

Combining the last two lemmas yields the corollary that $\operatorname{PoA}(d, s)$ is monotone increasing in $d$.

Corollary 1. The upper bound on $\operatorname{PoA}(d, s)$ from Theorem 1 is monotone increasing in $d$.

By Lemma 2, we can neglect the ceilings and replace $s$ by $\frac{d}{a}$ in the upper bound from Theorem 1 to get an upper bound on $\operatorname{PoA}\left(d,\left\lceil\frac{d}{a}\right\rceil\right)$. Furthermore, by Lemma 3, this upper bound has the largest value for $d \rightarrow \infty$. By computing this limit, we get:

Theorem 2. For Wardrop games with $(d, s)$-polynomial latency functions where $s=\left\lceil\frac{d}{a}\right\rceil$, we have

$$
\operatorname{PoA}\left(d,\left\lceil\frac{d}{a}\right\rceil\right) \leq \frac{a^{\frac{1}{a-1}} \cdot(a-1)}{\mathrm{e} \cdot \ln (a)}
$$

\subsection{Lower Bound}

For the lower bound, we consider an instance of a Wardrop game with $n=1$ player of traffic $w_{1}=1$. The network consists of two parallel edges $u$ and $\ell$ from node $s_{1}$ to node $t_{1}$. The latency functions are $f_{u}(x)=\alpha \cdot x^{s}$ and $f_{\ell}(x)=x^{d}$, where $\alpha \in \mathbb{R}_{>0}$ will be determined later. With a slight abuse of notation, let $\mathbf{z}=(z, 1-z)$ be a Wardrop equilibrium and let $\widehat{z}=(\widehat{z}, 1-\widehat{z})$ be the optimum strategy profile, where $z$ (resp. $\widehat{z}$ ) is the amount of traffic that is assigned to link $u$ in the Wardrop equilibrium (resp. optimum).

In the Wardrop equilibrium, the latency on both links is the same, so $z$ is the only positive solution to

$$
\alpha \cdot z^{s}=(1-z)^{d} .
$$

On the other hand, the optimum is defined by

$$
\widehat{z}:=\arg \min _{x \in[0,1]}\left\{\alpha \cdot x^{s+1}+(1-x)^{d+1}\right\},
$$

which yields that $\widehat{z}$ is the only positive solution to

$$
\alpha \cdot \frac{s+1}{d+1} \cdot \widehat{z}^{s}-(1-\widehat{z})^{d}=0 .
$$

Observe, that $z$ and $\widehat{z}$ are both dependent on $\alpha, s$ and $d$. If we can compute $z$ and $\widehat{z}$, then we can give a lower bound on the price of anarchy

$$
\operatorname{PoA}(d, s) \geq \frac{\mathrm{SC}(\mathrm{z})}{\mathrm{SC}(\widehat{z})}=\frac{\alpha \cdot z^{s+1}+(1-z)^{d+1}}{\alpha \cdot \widehat{z}^{s+1}+(1-\widehat{z})^{d+1}} .
$$

We can now further optimize this lower bound by choosing the best possible $\alpha$. 
The problem is that to determine $z$ and $\widehat{z}$, as we have to compute the root for polynomials with arbitrary degree as demanded for the equations (9) and (10). Numerical tests for all $(d, s)$-polynomials with $d \leq 30$ gives lower bounds that match the upper bounds from Theorem 1 up to some numeric precision. We have given example values for the factor $\alpha$ for polynomial latency functions up to a degree of 9 in Table 3.2, where reasonable. This is a strong indication that our lower bound might be matching for all $d \in \mathbb{N}$ and $s \in \mathbb{N}$ with $s \leq d$.

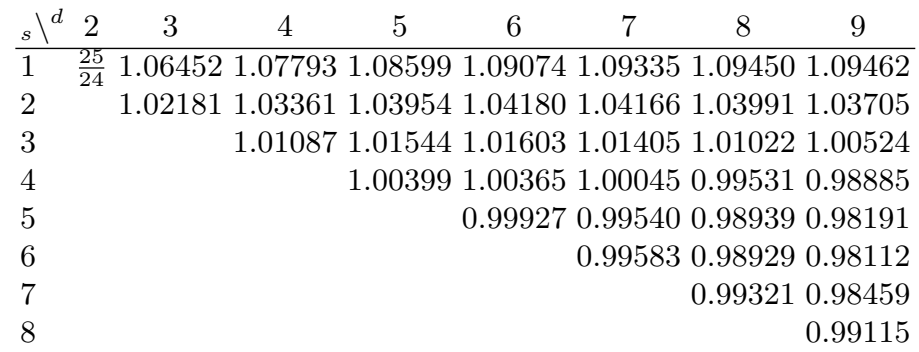

Table 2. Example values for $\alpha$, such that the lower bound is matching

\section{An Almost Matching Lower Bound for a Special Case}

We now show a lower bound on the price of anarchy for the case that $s=\frac{d}{2}$.

Set the constant factor $\alpha=1$. Then, the traffic on $u$ in the Wardrop equilibrium is the solution of the equation

$$
z^{\frac{d}{2}}=(1-z)^{d}
$$

which yields

$$
z=\frac{3}{2}-\frac{1}{2} \sqrt{5}
$$

We get that the social cost in the Wardrop equilibrium is

$$
\mathrm{SC}(\mathrm{z})=z^{\frac{d}{2}+1}+(1-z)^{d+1}=z^{\frac{d}{2}}=\left(\frac{3}{2}-\frac{1}{2} \sqrt{5}\right)^{\frac{d}{2}} .
$$

On the other hand, for the optimum we get that $\widehat{z}$ is the only positive root of

$$
\left(\frac{d}{2}+1\right) \widehat{z}^{\frac{d}{2}}-(d+1)(1-\widehat{z})^{d} .
$$

This root calculates to

$$
\widehat{z}=1+\frac{1-\sqrt{1+4\left(\frac{2 d+2}{d+2}\right)^{\frac{2}{d}}}}{2\left(\frac{2 d+2}{d+2}\right)^{\frac{2}{d}}} .
$$


The social cost in the optimum is

$$
\mathrm{SC}(\widehat{\mathbf{z}})=\widehat{z}^{\frac{d}{2}+1}+(1-\widehat{z})^{d+1}=\widehat{z}^{\frac{d}{2}} \cdot \frac{\frac{d}{2}+1+\frac{d}{2} \cdot \widehat{z}}{d+1} .
$$

We get

$$
\frac{\mathrm{SC}(\mathrm{z})}{\mathrm{SC}(\widehat{\mathrm{z}})}=\left(\frac{z}{\widehat{z}}\right)^{\frac{d}{2}} \cdot \frac{d+1}{\frac{d}{2}+1+\frac{d}{2} \cdot \widehat{z}}
$$

with limit

$$
\lim _{d \rightarrow \infty} \frac{\mathrm{SC}(\mathrm{z})}{\mathrm{SC}(\widehat{\mathrm{z}})}=\frac{1}{2^{\frac{1}{\sqrt{5}}}} \cdot \frac{4}{5-\sqrt{5}}>1.0614704
$$

Thus, for $d$ large enough, we have $\operatorname{PoA}\left(d, \frac{d}{2}\right) \geq 1.0614704$. This is slightly below the upper bound of $\operatorname{PoA}\left(d, \frac{d}{2}\right) \leq 1.0614756$. The same computations for $a=3$ yield a lower bound of $\operatorname{PoA}\left(d, \frac{d}{3}\right) \geq 1.159949$, which is again slightly below the upper bound of $\operatorname{PoA}\left(d, \frac{d}{3}\right) \leq 1.159983$. Note that for $a \geq 5$, we are again confronted with the problem of computing a general root.

\section{Conclusion}

In this paper, we have shown a general upper bound on the price of anarchy for Wardrop games with $(d, s)$-polynomial latency functions. We then proved monotonicity results on this upper bound and applied these to show that the price of anarchy is upper bounded by a constant, if $s$ is a constant fraction of $d$. As an example, for $s=\frac{d}{2}$ this upper bound is 1.0614756 . This implies that the price of anarchy does not only depend on the "steepness" of the latency functions, but rather on the presence of the lower monomials as the price of anarchy increases with the presence of lower monomials. Our discussion on lower bounds strongly indicates that our upper bound yields the exact value for the price of anarchy. However, the problem of finding a matching general lower bound that holds for all $(d, s)$-polynomials remains tantalizingly open.

\section{References}

1. S. Aland, D. Dumrauf, M. Gairing, B. Monien, and F. Schoppmann. Exact Price of Anarchy for Polynomial Congestion Games. Proceedings of the 23rd International Symposium on Theoretical Aspects of Computer Science (STACS'06), LNCS 3884, Springer Verlag, pages 218-229, 2006.

2. B. Awerbuch, Y. Azar, and A. Epstein. The Price of Routing Unsplittable Flow. In Proceedings of the 37th Annual ACM Symposium on Theory of Computing (STOC'05), pages 57-66, 2005.

3. M. Beckmann, C. B. McGuire, and C. B. Winsten. Studies in the Economics of Transportation. Yale University Press, 1956.

4. M. J. Beckmann. On the Theory of Traffic Flow in Networks. Traffic Quarterly, 21:109-116, 1967. 
5. G. Christodoulou and E. Koutsoupias. The Price of Anarchy of Finite Congestion Games. In Proceedings of the 37th Annual ACM Symposium on Theory of Computing (STOC'05), pages 67-73, 2005.

6. J. R. Correa, and A. S. Schulz and N. E. Stier-Moses. On the Inefficiency of Equilibria in Nonatomic Congestion Games. In Proceedings of the 11th Conference on Integer Programming and Combinatorial Optimization (IPCO'05), LNCS 3509, Springer Verlag, pages 167-181, 2005.

7. R. Cominetti, J. R. Correa, and N. E. Stier-Moses. Network Games With Atomic Players. In Proceedings of the 33rd International Colloquium on Automata, Languages, and Programming (ICALP'06), LNCS 4051, Springer Verlag, pages 525$536,2006$.

8. A. Czumaj and B. Vöcking. Tight Bounds for Worst-Case Equilibria. In Proceedings of the 13th Annual ACM-SIAM Symposium on Discrete Algorithms (SODA'02), pages 413-420, 2002. Also accepted to Journal of Algorithms as Special Issue of SODA'02.

9. M. Gairing, T. Lücking, M. Mavronicolas, and B. Monien. The Price of Anarchy for Polynomial Social Cost. Proceedings of the 29th International Symposium on Mathematical Foundations of Computer Science (MFCS'04), LNCS 3153, Springer Verlag, pages 574-585, 2004.

10. M. Gairing, T. Lücking, M. Mavronicolas, and B. Monien. The Price of Anarchy for Restricted Parallel Links. Parallel Processing Letters, 16(1):117-131, 2006.

11. M. Gairing, T. Lücking, B. Monien, and K. Tiemann. Nash Equilibria, the Price of Anarchy and the Fully Mixed Nash Equilibrium Conjecture. In Proceedings of the 32nd International Colloquium on Automata, Languages, and Programming (ICALP'05), LNCS 3580, Springer Verlag, pages 51-65, 2005.

12. M. Gairing, B. Monien, and K. Tiemann. Selfish Routing with Incomplete Information. In Proceedings of the 17th Annual ACM Symposium on Parallel Algorithms and Architectures (SPAA'05), pages 203-212, 2005.

13. M. Gairing, B. Monien, and K. Tiemann. Routing (Un-) Splittable Flow in Games with Player-Specific Linear Latency Functions. In Proceedings of the 33rd International Colloquium on Automata, Languages, and Programming (ICALP'06), LNCS 4051, Springer Verlag, pages 501-512, 2006.

14. E. Koutsoupias and C. H. Papadimitriou. Worst-Case Equilibria. Proceedings of the 16th International Symposium on Theoretical Aspects of Computer Science (STACS'99), LNCS 1563, Springer Verlag, pages 404-413, 1999.

15. M. Mavronicolas and P. Spirakis. The Price of Selfish Routing. In Proceedings of the 33rd Annual ACM Symposium on Theory of Computing (STOC'01), pages 510-519, 2001.

16. J. F. Nash. Non-Cooperative Games. Annals of Mathematics, 54(2):286-295, 1951.

17. A. C. Pigou. The Economics of Welfare. Macmillan and Company, 1920.

18. R. W. Rosenthal. A Class of Games Possessing Pure-Strategy Nash Equilibria. International Journal of Game Theory, 2:65-67, 1973.

19. T. Roughgarden. The Price of Anarchy is Independent of the Network Topology. Journal of Computer and System Sciences, 67(2):341-364, 2003.

20. T. Roughgarden. Selfish Routing and the Price of Anarchy. MIT Press, 2005.

21. T. Roughgarden and É. Tardos. How Bad Is Selfish Routing? Journal of the ACM, 49(2):236-259, 2002.

22. V. Vazirani. Approximation Algorithms. Springer Verlag, 2001.

23. J. G. Wardrop. Some Theoretical Aspects of Road Traffic Research. In Proceedings of the Institute of Civil Engineers, Pt. II, Vol. 1, pages 325-378, 1952. 